
tomateiro. Horticultura Brasileira 25: 387-391.

\title{
Fibra de coco e resíduo de algodão para substrato de mudas de tomateiro
}

\author{
Cândido A da Costa; Sílvio J Ramos; Regynaldo A Sampaio; Denílson O Guilherme; Luiz Arnaldo \\ Fernandes
}

UFMG - ICA, C. Postal 135, 39494-006 Montes Claros-MG; candido-costa@ufmg.br; sjramos2003@yahoo.com.br; rsampaio@ufmg.br; doliveiraguilherme@yahoo.com.br; larnaldo@ufmg.br

\section{RESUMO}

O efeito da mistura de fibra de coco (FC) e resíduo compostado de algodão (RA) foi avaliado na formação de substrato para produção de mudas de tomateiro Kada Gigante. O experimento foi conduzido em casa de vegetação no ICA da UFMG em Montes ClarosMG. Utilizou-se o delineamento experimental em blocos ao acaso, com quatro repetições. Os tratamentos avaliados foram: T1) Substrato comercial Hortimix ${ }^{\circledR}$; T2) 100\% massa seca de resíduo algodão proveniente da compostagem do resíduo da industria têxtil (RA); T3) $20 \%$ FC+80\% RA; T4) 40\% FC+60\% RA; T5) 60\% FC+40\% RA; T6) $80 \%$ FC+20\% RA; T7) 100\% FC+\% RA. Foram avaliados o diâmetro do caule, a altura da planta, o índice de velocidade de emergência, a porcentagem de germinação, a massa fresca da parte aérea e a massa fresca da raiz, a massa seca da aérea seca e a massa da matéria seca da raiz. Observou-se que o aumento da proporção de fibra de coco verde em relação ao resíduo compostado de algodão proporcionou maior emergência, e índice de velocidade de emergência, entretanto, o diâmetro do caule, a altura da planta, a massa fresca e seca da parte aérea e do sistema radicular diminuíram com o aumento dessa proporção. O resíduo de algodão proveniente da compostagem do resíduo da industria têxtil, constitui-se em um material útil na produção de substratos comerciais.

Palavras-chave: Lycopersicum esculentum, composto orgânico, hortaliça.

\author{
ABSTRACT \\ Coconut fiber and cotton waste as substrate for tomato \\ seedlings
}

A greenhouse experiment was carried out to evaluate fiber mixture of coconut and cotton waste, to produce substrate for tomato seedling production (cultivar Kada Gigante). The experimental design was randomized blocks, four replications, with seven treatments: T1) Commercial Substrate Hortimix ${ }^{\circledR}$; T2) 0\% coconut dry fiber mass (FC) $+100 \%$ dry mass of cotton waste (RA); T3) $20 \%$ (FC) $+80 \%$ (RA); T4) $40 \%$ (FC)+60\% (RA); T5) $60 \%$ (FC)+40\% (RA); T6) $80 \%$ (FC)+20\% (RA); T7) 100\% (FC)+0\% (RA). The vigor was evaluated in terms of percentage and index of emergence, stem diameter, seedling height. Increasing the proportion of coconut fiber to cotton waste ratio resulted in an increase of percentage and index of emergence; however, the stem diameter, seedling height, shoot and root fresh and dry mass decreased. The residue of cotton proceeding from the compostage of residues of the textil factory, constitutes in a useful material in the commercial substrate production.

Keywords: Lycopersicum esculentum, organic compost, vegetable.

(Recebido para publicação em 20 de abril de 2006; aceito em 30 de agosto de 2007)

A caracterização de resíduos urbanos e rurais e a avaliação de seu aproveitamento como substrato agrícola pode ser uma importante alternativa para a reciclagem destes materiais. Segundo Souza (2001), substratos alternativos podem ser utilizados para a produção de mudas e para o cultivo de plantas. Backes \& Kämpf (1991), Flynn et al. (1995), Chong (1999) e Sainju et al. (2001) destacam a importância da utilização de matérias primas regionais, tais como casca de arroz, bagaço de cana, casca de pinus, lixo urbano, resíduo da produção de papel, fibra de coco e o resíduo de algodão proveniente do processamento da industria têxtil. A fibra de coco e o resíduo de algodão são amplamente encontrados no município de Montes Claros e importantes do ponto de vista econômico, social e ambiental.
Segundo Carrijo et al. (2002), a fibra de coco verde apresenta características favoráveis para o seu aproveitamento como substrato no cultivo de hortaliças, devido à longa durabilidade sem alteração de suas características físicas, pela possibilidade de esterilização, a abundância da matéria prima renovável e o baixo custo para o produtor. Silveira et al. (2002) observaram que em substrato de fibra de coco verde, embora ocorresse maior porcentagem de germinação $(90,63 \%)$ do que no substrato comercial $(67,18 \%)$, as mudas de tomate foram menos vigorosas do que no substrato comercial. Esse problema foi corrigido quando o pó de coco foi misturado ao húmus de minhoca. Segundo esses mesmos autores, a utilização de pó de coco verde como substrato reduziu o custo da produção de mudas de tomateiro em torno de 47\%, além de constituir um subproduto abundante da agroindústria do coco, de ampla disponibilidade e de baixo valor no mercado.

A utilização de resíduo de algodão na composição de substratos e, posteriormente adicionado ao solo no momento do plantio, pode ter um efeito imediato ou um efeito residual, de acordo com o grau de decomposição desse resíduo. Segundo Johnson \& Crawford (1993), a aplicação de resíduo agroindustrial não maturado ao solo, além de conduzir à imobilização microbiológica de nitrogênio, pode ainda provocar decréscimo na concentração de oxigênio no solo e inibição da germinação de sementes, interferindo negativamente no desenvolvimento de plantas.

O objetivo deste trabalho foi avaliar a utilização do resíduo de algodão compostado proveniente da indústria têxtil, juntamente com a fibra de coco 
Tabela 1. Atributos químicos e físicos dos diferentes materiais utilizados na produção dos substratos para o cultivo de tomate (Chemical and physical characteristics of materials utilized in the substrate composition for tomato seedlings). Montes Claros, ICA-UFMG, 2005.

\begin{tabular}{|c|c|c|c|}
\hline Determinação & $\begin{array}{l}\text { Substrato } \\
\text { comercial } \\
\text { Hortimix® }\end{array}$ & Fibra de coco & $\begin{array}{l}\text { Resíduo de } \\
\text { algodão }\end{array}$ \\
\hline Matéria orgânica ${ }^{1}\left(\mathrm{~g} / \mathrm{dm}^{3}\right)$ & 22,60 & 24,77 & 23,45 \\
\hline pH em água & 6,0 & 6,6 & 6,8 \\
\hline $\mathrm{N}$ total $^{2}(\mathrm{dag} / \mathrm{kg})$ & 1,83 & 0,56 & 1,80 \\
\hline P disponível ${ }^{3}$ (dag/kg) & 0,92 & 0,15 & 0,19 \\
\hline $\mathrm{K}^{3}(\mathrm{dag} / \mathrm{kg})$ & 0,39 & 1,15 & 1,17 \\
\hline $\mathrm{Ca}^{4}(\mathrm{dag} / \mathrm{kg})$ & 0,24 & 0,45 & 0,46 \\
\hline $\mathrm{Mg}^{4}(\mathrm{dag} / \mathrm{kg})$ & 0,89 & 0,20 & 0,74 \\
\hline $\mathrm{S}(\mathrm{dag} / \mathrm{kg})$ & 0,03 & 0,02 & 0,04 \\
\hline $\mathrm{Zn}^{5}(\mathrm{mg} / \mathrm{kg})$ & 16,1 & 12 & 8 \\
\hline $\mathrm{Cu}^{5}(\mathrm{mg} / \mathrm{kg})$ & 14,5 & 0,3 & 2,89 \\
\hline $\mathrm{Mn}(\mathrm{mg} / \mathrm{kg})$ & 45,1 & 14 & 15,24 \\
\hline densidade $\left(\mathrm{kg} \mathrm{dm}^{-3}\right)$ & 0,47 & 0,98 & 0,45 \\
\hline $\begin{array}{l}\text { capacidade de retenção de água } \\
\text { ( } \mathrm{g} \mathrm{H}_{2} 0 \cdot \mathrm{g}^{-1} \text { substrato) }\end{array}$ & 2,7 & 3,0 & 1,0 \\
\hline $\mathrm{C}^{-E^{6}}\left(\mathrm{dS} . \mathrm{m}^{-1}\right)$ & 1,80 & 2,18 & 8,40 \\
\hline
\end{tabular}

${ }^{1}$ Método da perda por irrigação (Kiehl, 1985); ${ }^{2}$ Método micro-Kjeldahl (Kiehl, 1985); ${ }^{3}$ Extrator Mehlich-1 (Defelipo \& Ribeiro, 1981); ${ }^{4}$ Extrator KCl 1mol/L (Defelipo \& Ribeiro, 1981); ${ }^{5}$ Extrator $\mathrm{HNO}_{3} / \mathrm{HClO}_{4}$; ${ }^{6}$ Extrato de saturação na relação 1:5 (Tedesco et al., 1995) ( ${ }^{1}$ method of the loose from irrigation (Kiehl, 1985); ${ }^{2}$ method micro-Kjeldahl (Kiehl, 1985); ${ }^{3}$ Extractor Mehlich-1 (Defelipo \& Ribeiro, 1981); ${ }^{4}$ Extractor KCl 1mol/L (Defelipo \& Ribeiro, 1981); ${ }^{5}$ Extractor $\mathrm{HNO}_{3} / \mathrm{HClO}_{4},{ }^{6}$ Extract of saturation on the relation 1:5 (Tedesco et al., 1995)).

verde, como substrato para a produção de mudas de tomateiro.

\section{MATERIAL E MÉTODOS}

O experimento foi conduzido em casa de vegetação do Instituto de Ciências Agrárias da UFMG em Montes Cla-
ros-MG. Utilizou-se o tomate (Lycopersicum esculentum Mill, cultivar Kada Gigante.

Avaliou-se os tratamentos $\left.\mathrm{T}_{1}\right)$ Substrato comercial Hortimix ${ }^{\circledR}$ (composto pela casca de pínus bioestabilizada, vermiculita, calcário dolomítico e fertilizante NPK); $\mathrm{T}_{2}$ ) 0\% massa seca de fibra de coco $+100 \%$ massa seca de resíduo de algodão proveniente da indústria têxtil (resíduo de algodão); $\mathrm{T}_{3}$ ) 20\% massa seca de fibra de coco $+80 \%$ massa seca de resíduo de algodão; $T_{4}$ ) 40\% massa seca de fibra de coco $+60 \%$ massa seca de resíduo de algodão; $\mathrm{T}_{5}$ ) 60\% massa seca de fibra de coco +40 massa seca de resíduo de algodão; $\mathrm{T}_{6}$ ) 80\% massa seca de fibra de coco $+20 \%$ massa seca de resíduo de algodão; $\mathrm{T}_{7}$ ) 100\% massa seca de fibra de coco $+0 \%$ massa seca de resíduo de algodão. As características químicas dos materiais usados na produção dos substratos encontram-se na tabela 1.

A semeadura manual foi em bandejas de poliestireno expandido, contendo 128 células, colocando-se uma semente no centro de cada célula da bandeja. Foi feita irrigação intermitente com microaspersor nebulizador Fogger Tietze, bocal azul de $7 \mathrm{~L} \mathrm{~h}^{-1}$, pressão de 40 mca acoplado a uma válvula antigotejo Tietze de baixa pressão, sendo os intervalos de acordo com as condições ambientais do local. O delineamento experimental foi de blocos casualizados, com quatro repetições. A unidade experimental foi constituída por 16 plantas, sendo avaliados o diâmetro da base do hipocótilo (D), a altura da planta (A), o índice de velocidade de emergência (IVE), a porcentagem de germinação $(G)$, a massa aérea fresca (MPAF) e da raiz (MRF), e, a massa aérea seca (MPAS) e da raiz (MRS). A

Tabela 2. Valores médios obtidos de Diâmetro (D), Altura (A), Índice de velocidade de emergência (IVE), Germinação (G), Massa fresca da parte aérea (MFPA), Massa fresca do sistema radicular (MFSR), Massa seca da parte aérea (MSPA), Massa seca do sistema radicular (MSSR) em mudas de tomate (Lycopersicum esculentum M.) cultivar Kada Gigante, produzidas em diferentes substratos (mean values of diameter (D), height (A), emergency velocity index (IVE), germination (G), fresh mass of the aerial part (MFPA), fresh mass of roots (MFSR), dry mass of aerial part (MSPA), dry mass of roots (MSSR) on tomato seedlings, cv. Kada Gigante, produced in various substrates). Montes Claros, ICA-UFMG, 2005.

\begin{tabular}{|c|c|c|c|c|c|c|c|c|}
\hline Substratos & $\mathrm{D}(\mathrm{mm})$ & $A(\mathrm{~cm})$ & IVE (dias) & G (\%) & MPAF (g) & MPAS (g) & MSRF (g) & MSRS (g) \\
\hline $0 \% \mathrm{FC}+100 \% \mathrm{RA}$ & $1,32^{*}$ & $6,81^{*}$ & $2,06^{*}$ & $36,06^{*}$ & $0,1659^{*}$ & $0,0291^{*}$ & $0,1246^{*}$ & $0,0112^{*}$ \\
\hline $20 \% \mathrm{FC}+80 \% \mathrm{RA}$ & $0,94^{*}$ & $3,25^{*}$ & $2,42^{\text {ns }}$ & $78,12^{\text {ns }}$ & $0,0675^{*}$ & $0,0106^{*}$ & $0,0593^{*}$ & $0,0045^{*}$ \\
\hline $40 \% \mathrm{FC}+60 \% \mathrm{RA}$ & $0,88^{*}$ & $2,64^{*}$ & 2,64 ns & 89,06 ns & $0,0373^{*}$ & $0,0051^{*}$ & $0,0195^{*}$ & $0,0034^{*}$ \\
\hline $60 \% \mathrm{FC}+40 \% \mathrm{RA}$ & $0,93^{*}$ & $2,46^{*}$ & 2,35 ns & 85,93 ns & $0,0441^{*}$ & $0,0046^{*}$ & $0,0180^{*}$ & $0,0015^{*}$ \\
\hline $80 \% \mathrm{FC}+20 \% \mathrm{RA}$ & $0,94^{*}$ & $2,46^{*}$ & 2,32 ns & 87,43 ns & $0,0353^{*}$ & $0,0037^{*}$ & $0,0229^{*}$ & $0,0023^{*}$ \\
\hline $100 \% \mathrm{FC}+0 \% \mathrm{RA}$ & $0,91^{*}$ & $2,37^{*}$ & 2,39 ns & 92,18 ns & $0,0332^{*}$ & $0,0044^{*}$ & $0,0256^{*}$ & $0,0019^{*}$ \\
\hline substrato comercial Hortimix $®$ & 1,78 & 9,01 & 2,96 & 90,62 & 0,7913 & 0,0622 & 0,6242 & 0,0385 \\
\hline (DMS) & 1,08 & 0,43 & 0,71 & 25,42 & 0,081 & 0,071 & 0,225 & 0,021 \\
\hline
\end{tabular}

*Difere estatisticamente pelo teste de Dunnett a 5\% de probabilidade na comparação com o substrato comercial; FC = Fibra de coco; RA = Resíduo de algodão compostado proveniente da industria têxtil (statistical difference, Dunnett test, 5\%, compared to the commercial substrate; FC = coconut fiber; RA = residue from composted cotton of the textil factory). 


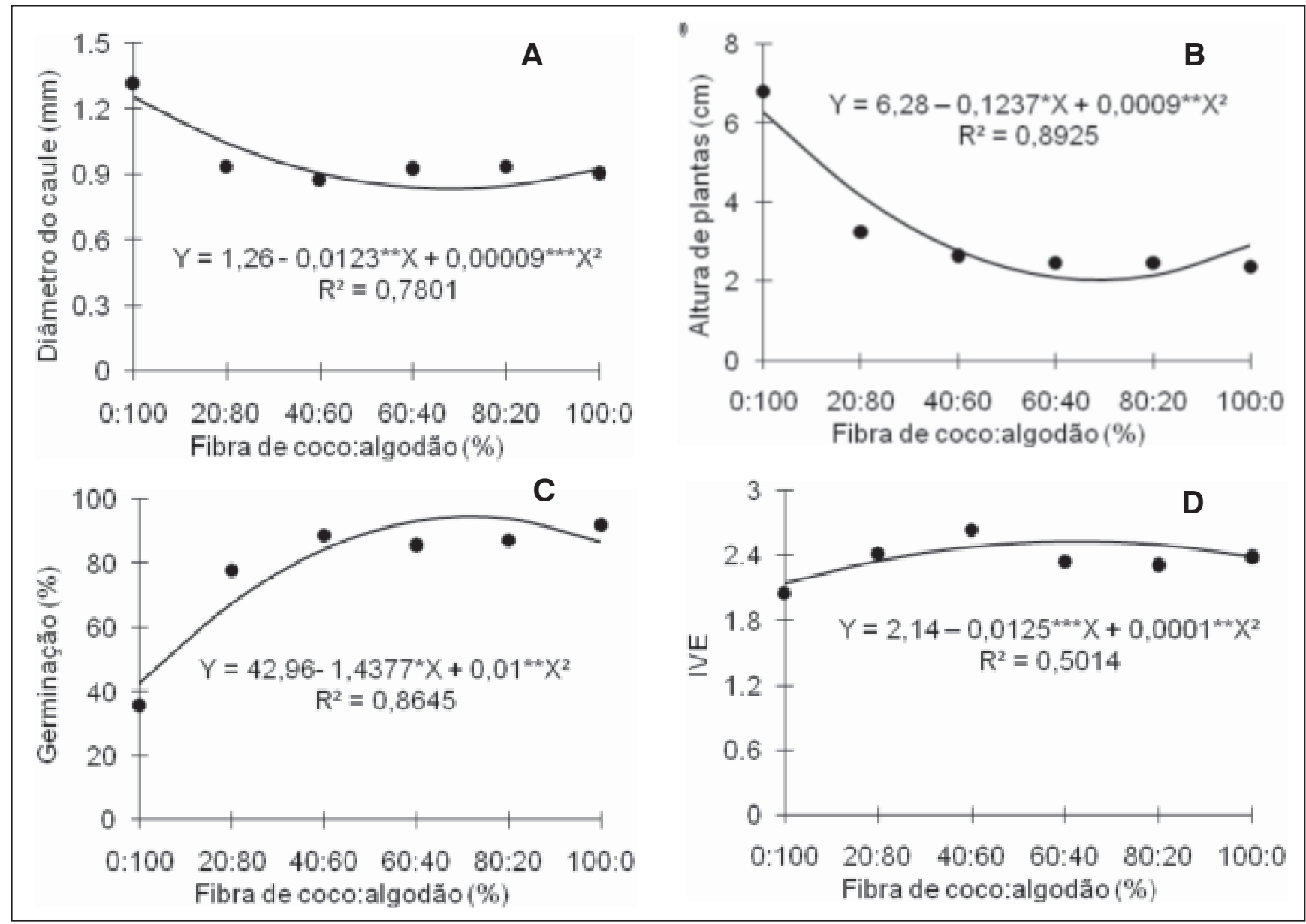

Figura 1. Diâmetro (A), Altura (B), Germinação (C), Indice de Velocidade de Emergência (IVE) (D) de plântulas do tomateiro em função da porcentagem de fibra de coco (diameter (A), height (B), germination (C), emergency velocity index (IVE) (D) of tomato plantlets as a result of the percentual of coconut fiber). Montes Claros, ICA-UFMG, 2005.

*, **, *** Significativos a 5, 1 e $0,1 \%$ de probabilidade, respectivamente, pelo teste $\mathrm{t}(*, * *, * * *$ significant at $5 ; 1$ and $0,1 \%$ probability, respectively, $\mathrm{t}$ test).

germinação foi avaliada aos 20 dias após a semeadura, considerando-se germinadas as sementes que emitiram a radícula. A altura da planta, medida da base do hipocótilo até o ápice da muda, e o diâmetro do hipocótilo na região da base, foram avaliados aos 28 dias após a semeadura. Os dados obtidos foram submetidos à análise de variância e as médias das proporções de fibra de coco e de resíduo de algodão foram comparadas com as do substrato comercial até $5 \%$ de probabilidade pelo teste de Dunnett. As médias referentes às proporções de fibra de coco e de resíduo foram ajustadas a modelos de regressão até $10 \%$ de probabilidade pelo teste t.

\section{RESULTADOS E DISCUSSÃO}

Observaram-se diferenças significativas entre o substrato constituído de fi- bra de coco e de resíduo de algodão e o substrato comercial $\left(\right.$ Hortimix $^{\circledR}$ ) para todas as variáveis estudadas, com exceção apenas do índice de velocidade emergência e da percentagem de germinação. O substrato comercial proporcionou os melhores resultados (Tabela 2), os quais podem estar relacionados à maior disponibilidade de nutrientes, principalmente fósforo, e à retenção de umidade, favorecendo o processo germinativo e o desenvolvimento pósseminal (Tabela 1) (Mayer \& PoljakoffMayber, 1989; Figliola, 1993). Desempenhos melhores do substrato comercial em relação a substratos alternativos foram também observados por Bezerra et al. (2002), Ledo et al. (2002) e Oliveira et al. (2004).

O aumento da proporção de fibra de coco em relação ao resíduo compostado de algodão proporcionou maior por- centagem de germinação de sementes, e índice de velocidade de emergência, possivelmente, relacionado à maior condutividade elétrica do resíduo de algodão. Entretanto, o diâmetro do caule, a altura da planta, a massa fresca e seca da parte aérea, a massa fresca e seca do sistema radicular, diminuíram com o aumento da fibra de coco (Tabela 2). Tal fato pode ser atribuído a uma possível limitação de nutrientes neste resíduo, conforme também observado por Rosa et al. (2001).

Houve influência das proporções de fibra de coco e do resíduo de algodão nas características das mudas (Figura 1). O diâmetro do caule apresentou tendência de diâmetro mínimo de $0,83 \mathrm{~mm}$ com 68,33\% de fibra coco na relação com resíduo de algodão. Por outro lado, valores abaixo de 68,33\% da proporção de fibra de coco no substrato, promove- 

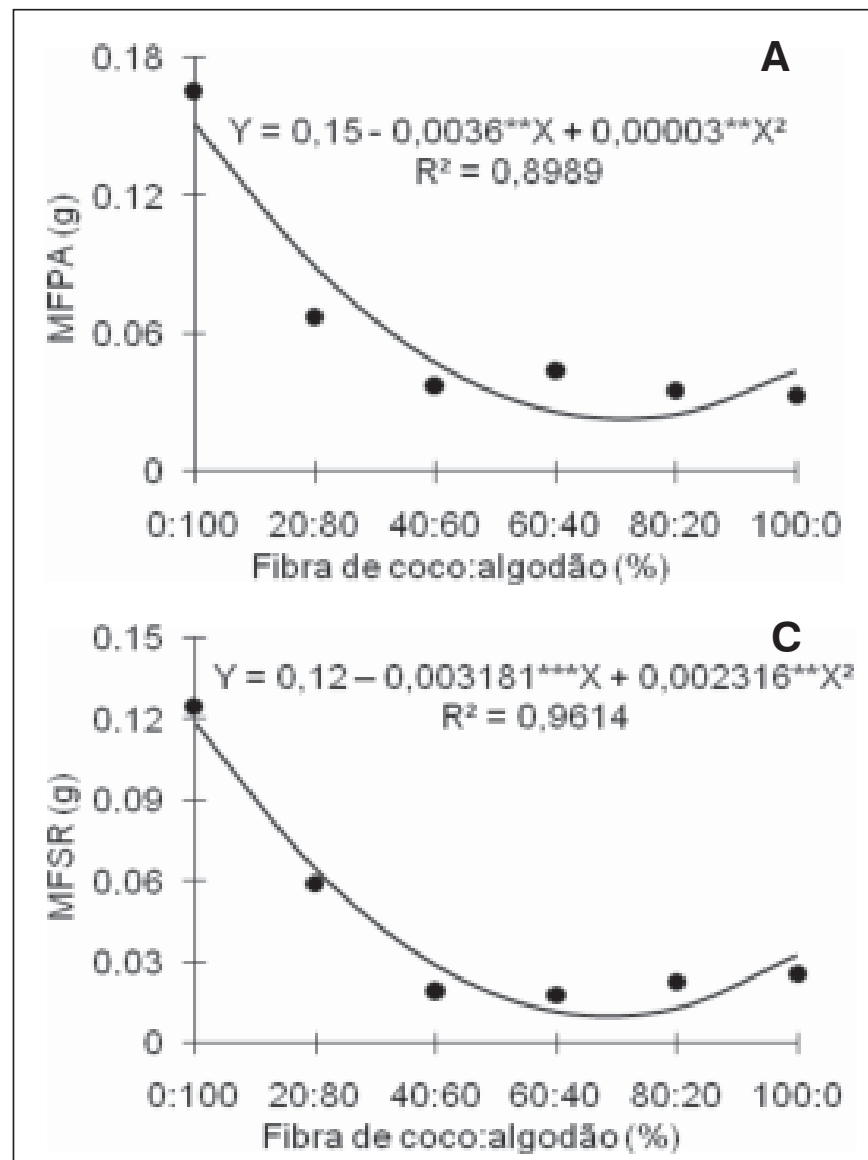
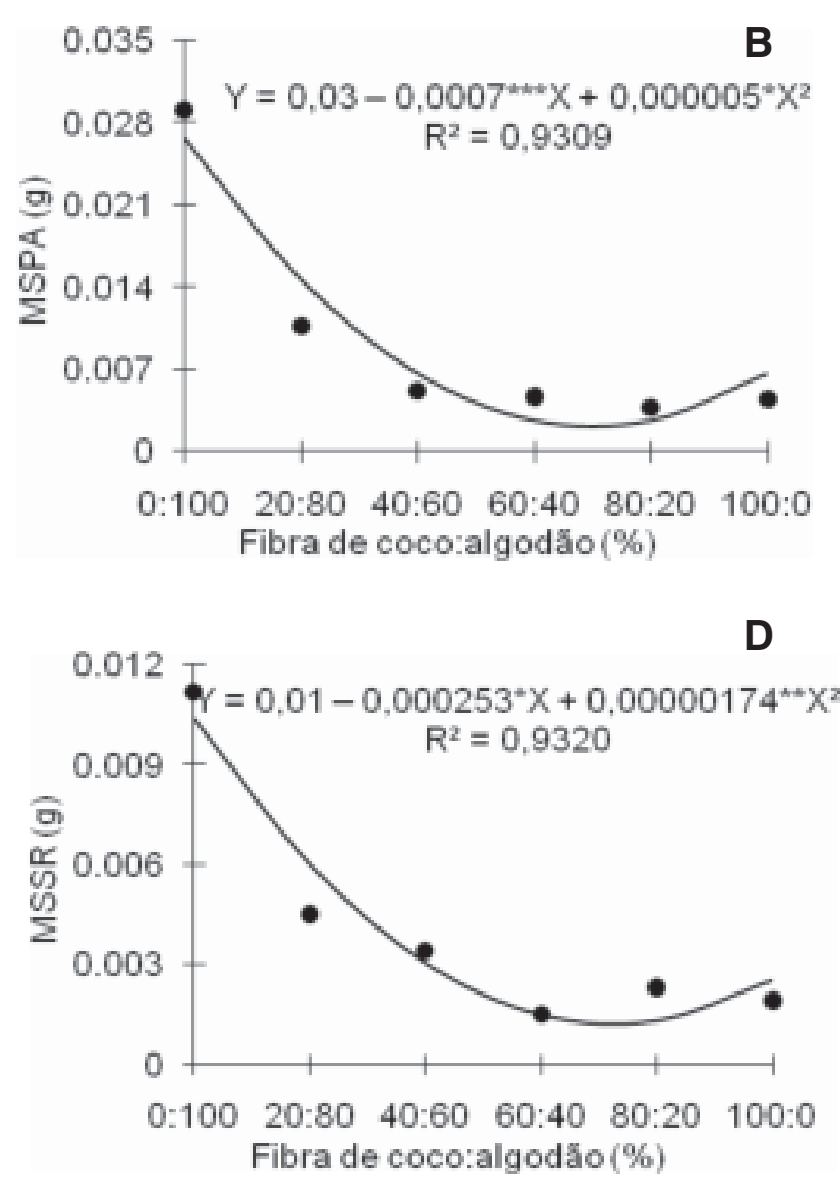

Figura 2. MFPA (A), MSPA (B), MFSR (C), MSSR (D) de plântulas do tomateiro em função da porcentagem de fibra de coco (MFPA (A), MSPA (B), MFSR (C), MSSR (D) of tomato plantlets as a result of the percentual of coconut fiber). Montes Claros, ICA-UFMG, 2005. $*, * *, * * *$ Significativos a 5,1 e $0,1 \%$ de probabilidade, respectivamente, pelo teste $\mathrm{t}(*, * *, * * *$ significant at $5 ; 1$ and $0,1 \%$ probability, respectively, $\mathrm{t}$ test).

ram aumento deste diâmetro, tendo uma produção melhor quando o resíduo de algodão foi utilizado puro (Figura 1A). O melhor desenvolvimento no resíduo de algodão das mudas pode ser explicado pela melhor disponibilidade de nutrientes (Tabela 1).

A resposta da altura de mudas de tomateiro em relação à mistura da fibra de coco com resíduo de algodão foi quadrática (Figura 1B), obtendo-se altura mínima de $2,02 \mathrm{~cm}$, com a relação de $68,72 \%$ de fibra de coco. Esta variável apresentou maiores valores com o aumento da proporção de resíduo de algodão no substrato. É possível que a fibra de coco não tenha proporcionado boas condições de aeração e suprimento de nutrientes para as plantas devido a lenta decomposição e liberação de nutrientes (Tabela 1). Estes resultados não concordam com Gadelha et al. (2000), onde estes observaram efeito positivo do uso de substratos contendo fibra de coco misturado à casca de arroz carbonizada, pau turfoso e húmus de minhoca, na produção de mudas de espécies como cajueiro anão-precoce.

Constatou-se, com base no ajuste da regressão, germinação máxima de $94,63 \%$ com a relação de $71,88 \%$ de fibra de coco (Figura 1C). Proporções de resíduo de algodão acima de 30\% na composição dos substratos promoveram diminuição da porcentagem de germinação. Tal fato pode estar relacionado à menor disponibilidade de água deste substrato, uma vez que o resíduo de algodão retém menos água comparado aos outros substratos (Tabela 1). Minami (1995) cita que o substrato é o componente mais sensível e complexo do sistema de produção de mudas, pois, qualquer variação na sua composição pode alterar o processo final de produção de mudas, desde a não germinação de sementes até o desenvolvimento irregular das plantas.

O índice de velocidade de emergência (IVE) também apresentou ajuste quadrático em relação à proporção fibra de coco e resíduo de algodão, com destaque para o maior índice de 2,53 na proporção de $62,50 \%$ de fibra de coco (Figura 1D).

Com relação às massas aérea fresca e seca, os resultados expressaram resposta quadrática. Os menores valores obtidos para estas variáveis foram, respectivamente, 0,0434 e $0,0022 \mathrm{~g}$, nas proporções de fibra de coco de $40 \%$ e $30 \%$ de resíduo de algodão (Figura 2A e 2B). Portanto, constatou-se aumento do crescimento e, conseqüentemente, das massas aérea seca e fresca, a partir de 30 a 40\%, respectivamente, de resí- 
duo de algodão na constituição do substrato.

As massas fresca e seca do sistema radicular apresentaram ajuste quadrático em relação às diferentes proporções de fibra de coco e resíduo de algodão. Os menores valores obtidos para essas variáveis foram com 80 e $75 \%$, respectivamente, de fibra de coco (Figura 2C e 2D). Ficou evidente, portanto, a diminuição da massa da raiz seca com o incremento da proporção de fibra de coco em relação ao resíduo de algodão. Segundo Rosa et al. (2001), uma das principais limitações do uso de fibra de coco como substrato é a baixa disponibilidade de nutrientes. Isso é explicado pela lenta decomposição do material orgânico em função da baixa relação $\mathrm{C} / \mathrm{N}$. Entretanto, Kämpf \& Fermino (2000) afirmam que o efeito negativo da fibra de coco no substrato sobre as características das plantas pode estar relacionado a uma possível presença de taninos solúveis que, quando muito concentrados na fibra de coco, são fitotóxicos e inibem o crescimento das raízes, afetando o crescimento das plantas.

O substrato comercial (Hortimix ${ }^{\circledR}$ ) apresentou resultados superiores aos substratos contendo resíduos de algodão e fibra de coco.

Para a germinação e índice de velocidade de germinação, os melhores resultados de substratos contendo resíduo de algodão e fibra de coco, foram obtidos quando se utilizou a proporção de 65\% de resíduo de algodão.

Em relação ao diâmetro do caule, altura da planta e massas fresca e seca da raiz e da parte aérea, os melhores re- sultados de substratos contendo resíduo de algodão e fibra de coco, fora obtidos quando se utilizou $100 \%$ de resíduo de algodão.

\section{REFERÊNCIAS}

BACKES MA; KÄMPF AN. 1991. Substratos à base de composto de lixo urbano para a produção de plantas ornamentais. Pesquisa Agropecuária Brasileira 26: 753-758.

BEZERRA AME; MOMENTÉ VG; ARAÚJO EC; MEDEIROS FILHO S. 2002. Germinação e desenvolvimento de plântulas de melãode-são-caetano em diferentes ambientes e substratos. Revista Ciência Agronômica 33: 39-44.

CARRIJO OA; LIZ RS; MAKISHIMA N. 2002. Fibra da casca do coco verde como substrato agrícola. Horticultura Brasileira 20: 533-535.

CHONG C. 1999. Experiences with the utilization of wastes in nursery potting mixes and field soil amendments. Canadian Journal of Plant Science 79: 139-148.

DEFELIPO BV; RIBEIRO AC. 1981. Análise química do solo (metodologia). Viçosa, UFV, Impr. Univ. 17p. (Boletim de Extensão, 29).

FIGLIOLA MB; OLIVEIRA EC; PIÑARODRIGUES FCM. 1993. Análise de sementes. In: AGUIAR IB; PIÑA-RODRIGUES FCM; FIGLIOLA MB. (coord). Sementes florestais tropicais. Brasília: ABRATES, 350p.

FLYNN RP; WOOD CW; GUERTAL EA. 1995. Lettuce response to composted broiler litter as a potting substrate component. Journal of American Society for Horticultural Science 6: 964-970.

GADELHA JWR; CORRÊA MPF; CORREIA D; ROSSETTTI AG; RIBEIRO EM. 2000. Efeitos de substratos e da idade do porta-enxerto na formação de mudas de cajueiro anão precoce (Anacardium occidentale L.) em tubetes. In: CONGRESSO BRASILEIRO DE FRUTICULTURA, 16., 2000, Fortaleza. Anais... Fortaleza: SBF, p.175.

JOHNSON GE; CRAWFORD SL. 1993. Evaluating compost quality. Resource Recycling p.50-54.
LEDO AS; MEDEIROS FILHO S; LEDO FJS; ARAÚJO EC. 2002. Efeito do tamanho da semente, do substrato e pré-embebição na germinação de sementes de pupunha. Revista Ciência Agronômica 33: 29-32.

KÄMPF AN; FERMINO MH. 2000. Seleção de materiais para uso como substrato.Substratos para plantas: a base da produção vegetal em recipientes. In: KAMPF AN; FERMINO MH. (Ed.). Substratos para plantas: a base da produção vegetal em recipientes. Porto Alegre: Gênesis, p.139-145.

KIEHL EJ. 1985. Fertilizantes orgânicos. São Paulo, Ceres, 492p.

MINAMI K. 1995. Fisiologia da produção de mudas. São Paulo: T.A. Queiroz, 129 p.

MAYER AC; POLJAKOFF-MAYBER A. 1989. The germination of seeds. London: Pergamon Press, 270p.

OLIVEIRA GB; MOTAWF; MAGALHÃES VR; ALVES FG; SILVEIRA EKCP; TARCHETTI GP. 2004. Produção de mudas de tomateiro rasteiro com a utilização de substratos alternativos ao produto comercialmente utilizado. Horticultura Brasileira 22; Suplemento CDROM. Trabalho apresentado no $44^{\circ}$ Congresso Brasileiro de Olericultura.

ROSA MF; SANTOS FJS; MONTENEGRO AAT; ABREU FAP; CORREIA D; ARAÚJO FBS. 2001. Caracterização do pó da casca de coco verde usado como substrato agrícola. Fortaleza: Embrapa-CNPAT, 6 p. (Comunicado Técnico, 54).

SAINJU UM; RAHMAN S; SINGH BP. 2001. Evaluating hairy vetch residue as nitrogen fertilizer for tomato in soilless medium. HortScience 36: 90-93.

SILVEIRA EB; RODRIGUES VJLB; GOMES AMA; MARIANO RLR; MESQUITA JCP. 2002. Pó de coco como substrato para produção de mudas de tomateiro. Horticultura Brasileira 20: 211-216.

SOUZA FX. 2001. Materiais para formulação de substratos na produção de mudas e no cultivo de plantas envasadas. Fortaleza: EmbrapaCNPAT, 21 p. (Documentos, 43).

TEDESCO MJ; GIANELLO C; BISSANI CA; BOHNEN H; VOLKWEISS SJ. 1995. Análise de solo, plantas e outros materiais. UFRGS: Porto Alegre, 174p. 